\title{
Uniform and Amorphous Rifampicin Microspheres Obtained by Freezing Induced LLPS during Lyophilization
}

\author{
Chun Liu ${ }^{1,3}$, Chao Kong ${ }^{1,3}$, Guoliang $\mathrm{Wu}^{1,3}$, Junhao Zhu ${ }^{2,3}$, Babak Javid ${ }^{2,3}$, Feng Qian ${ }^{1,3 *}$ \\ ${ }^{1}$ Department of Pharmacology and Pharmaceutical Sciences, ${ }^{2}$ Department of Basic Medical \\ Sciences, and ${ }^{3}$ Collaborative Innovation Center for Diagnosis and Treatment of Infectious \\ Diseases, School of Medicine, Tsinghua University, Beijing, 100084, P.R.China
}

*To whom correspondence should be addressed:

Email: qianfeng@biomed.tsinghua.edu.cn, 86-10-62794744

Manuscript for International Journal of Pharmaceutics

(C) 2015. This manuscript version is made available under the Elsevier user license http://www.elsevier.com/open-access/userlicense/1.0/ 


\begin{abstract}
By lyophilization of rifampicin (RIF) solution in TBA/water with various solvent compositions, uniform and amorphous rifampicin (RIF) microspheres were produced. Using 55\% TBA solution, the obtained RIF microspheres have a mono-dispersive size distribution with diameters range from 1-3 $\mu \mathrm{m}$. The RIF microspheres are found to be amorphous by $\mathrm{x}$-ray diffraction, and are expected to dissolve much faster than the crystalline RIF upon inhalation. Mechanistic investigation revealed that the amorphous RIF microspheres were formed due to liquid-liquid phase separation (LLPS) occurred during the freezing of the TBA/water solution. We also observed that the RIF microspheres can be readily phagocytized by activated THP-1 cells within 15 minutes. The suitable size distribution, high solubility, and readiness for phagocytosis by macrophages, all suggest that the lyophilized amorphous RIF microspheres could be potentially used as an anti-tuberculosis inhalation therapy. In addition, similar process was used to lyophilize TBA/water solutions of several other drugs, including rifaximin, rifapentine, paclitaxel, and isoniazid. We found that for drugs with appropriate physiochemical properties, such as paclitaxel and rifaximin, mono-dispersive microspheres could be obtained as well, which demonstrated that freezing induced LLPS could be utilized as a novel particle engineering methodology to produce drug microspheres by lyophilization.
\end{abstract}

Keyword: rifampicin, lyophilization, liquid-liquid phase separation (LLPS), inhalation, tuberculosis 


\section{Abbreviations:}

LLPS: liquid-liquid phase separation

RIF: rifampicin

TBA: tert-butyl alcohol

POM: polarized microscope

SEM: scanning electron microscopy

DLS: dynamic light scattering

PXRD: powder x-ray diffraction

Mtb: mycobacterium tuberculosis

TB: tuberculosis

AMФ: alveolar macrophage

DPI: dry powder inhaler

DNA: deoxyribonucleic acid

RNA: ribonucleic acid

ATCC: American type culture collection

FBS: fetal bovine serum

PMA: phorbol myristate acetate

API: active pharmaceutical ingredient

PBS: phosphate buffered saline 


\section{Introduction}

Mycobacterium tuberculosis (Mtb) primarily infects pulmonary alveoli, multiplies in alveolar macrophages (AMФs), and could avoid fusion with lysosome in the form of phagosome vesicles (Muttil et al., 2009). After growing in the lungs for 2 to 12 weeks, the host cell-mediated immunity develops, granulomas with decreased blood vessels are formed and surrounded by thick calcified fibrous layer (Russell, 2007), which could prevent conventional oral drug therapy from achieving sufficient therapeutic concentration in the lesions. In oral drug therapy, multiple co-administered anti-TB drugs, high dose, and long treatment duration (6-24 months) are usually required, which are often associated with poor patient compliance, serious side effects, and acquired drug resistance (Chhabria et al., 2009; Dorman and Chaisson, 2007; Ginsberg, 2010). Inhaled anti-TB drugs, with suitable pharmaceutical properties, might be able to target the AMФs in the deep lung, where Mtb resides, and significantly increase the therapeutic effect, decrease side effect, shorten treatment duration, and prevent drug resistance (Blasi et al., 2009; Misra et al., 2011; Muttil et al., 2009; Rastogi et al., 2006).

To efficiently target the Mtb residing AMФs in the deep lung, inhaled dry powders with the following properties are highly desirable: 1), Aerodynamic diameter of 1-3 $\mu \mathrm{m}$, with good powder properties to be dispersed from a dry powder inhaler (DPI) and to reach the deep lung (Chow et al., 2007; Shoyele and Cawthorne, 2006); 2), Geometric diameter between 0.5-6 $\mu$ m, with appropriate hydrophobicity, surface charge, and surface functional groups to enhance phagocytosis by AMФs (Champion et al., 2008; Makino et al., 2003; Walkey et al., 2012; Wattendorf et al., 2006; Yoo et al., 2011).

The most commonly used commercial process to produce inhaled dry powder is jet-milling (Seville et al., 2007; Shoyele, 2008). However, as a mechanical attrition process, jet milling has 
poor control over particle size, density, morphology, and surface properties, all critically needed for $А M \Phi$-targeted drug delivery. The viability of jet milling is also heavily dependent on the mechanical property of the drug particle. Spray drying is another pharmaceutical process widely used by many research groups (Chow et al., 2007; Seville et al., 2007) to generate micro-sized particles for pulmonary drug delivery. However, to commercialize a spray-drying process to manufacture particles $<10 \mu \mathrm{m}$ for inhalation products, significant engineering expertise and heavy capital investment (e.g., recovery of organic solvents, etc.) are needed. Spray dried powder is typically poorly flowing and electrostatic, which makes the powder filling step very challenging. Besides these two most commonly used process, some emerging process technologies have also been explored for particle engineering purpose, such as low pressure spraying method (Enayati et al., 2010; Nangrejo et al., 2008), and surface modifying microfluidic processes (Kucuk et al., 2014)

Lyophilization or freeze-drying is a pharmaceutical process commonly used to convert drug solution into solid form to achieve long term stability (L. Remmele et al., 2012; Wang, 2000). Lyophilization could occur directly in the container of the final dosage form, avoiding the difficulty of filling the electrostatic and poorly flowing powder into various containers. Typically, lyophilization is not perceived as a particle engineering tool. However, we have previously showed that particles with well controlled particle properties could be synthesized by lyophilization process, if appropriate lyophilization solvents and process conditions are utilized, based on the mechanistic understanding of the phase behavior of all involved materials and the property of the drug substance (de Waard et al., 2008; Overhoff et al., 2007; Qian et al., 2008).

In this study, we prepared RIF microparticles by lyophilization process for potential inhalation therapy. RIF (Fig. 1) is a first-line anti-TB drug which lays the foundation for the current 6- 
month anti-tuberculosis regimens in combination with pyrazinamide, isoniazid and ethambutol (Mitchison, 2000). The anti-tuberculosis effect of rifampicin is due to its high affinity binding to, and inhibition of the activity of the bacterial DNA-dependent RNA polymerase (Hartmann et al., 1967). Some research has demonstrated that local high concentration of rifampicin could facilitate its diffusion into the alveolus region, which further favors sterilization in a shorter term (Diacon et al., 2007; Ruslami et al., 2007). While with oral administration, the poor RIF delivery efficiency into the lungs due to its first pass metabolism, poor solubility and subsequent low bioavailability limit its fast action, RIF administration through pulmonary inhalation could potentially exert rapid and potent therapy directly to the infectious sites (i.e. alveoli in deep lung) and generate high local drug concentration around the lesions to kill bacteria immediately, thereby, either greatly reducing the frequency of drug administration or treatment duration or both and thus improve patient compliance.

In this study, we observed the formation of amorphous and uniform RIF microspheres upon a one-step lyophilization process of TBA/water solution. We concluded that LLPS of the RIF TBA/water solution during freezing was the underlying mechanism contributing to the microsphere formation. Furthermore, we also observed similar microsphere formation when lyophilizing TBA/water solution of several other drugs, which implies that lyophilization with LLPS could be utilized as a novel particle engineering tool for drugs with suitable physiochemical properties.

\section{Materials and Methods}

\subsection{Materials}


Rifampicin (MW 822.94, purity: 98\%) was purchased from Beijing Ouhe Technology CO., LTD (Beijing), tert-butyl alcohol (TBA) was purchased from Xilong Chemical CO., LTD (Shantou, Guangzhou). Ultrapure deionized water was used during the experiment. The THP-1 cell from ATCC was cultured in medium RPMI 1640 (Gibco, Life) supplemented with 10\% fetal bovine serum (FBS) (Gibco, Life) and 1\% penicillin and streptomycin (Gibco, Life).

\subsection{Methods}

\subsubsection{Measurement of the solubility of crystal form RIF in TBA/water cosolvent}

Excess of RIF was added into various percentages of TBA solution $(0,30 \%, 35 \%, 40 \%, 45 \%$, $50 \%$, and $55 \%, 60 \%, 65 \%, 70 \%, 80 \%, 100 \%$ of $\mathrm{TBA} / \mathrm{water}, \mathrm{w} / \mathrm{w})$ in sealed glass vials and stirred under $37^{\circ} \mathrm{C}$ bath for 12 hours and then standing for 24 hours. After centrifuging and filtering supernatant through $0.22 \mu \mathrm{m}$ filter, the absorbance of RIF was measured by UV4802 spectrophotometer (Unico, Shanghai) at $474 \mathrm{~nm}$ and the concentration of RIF was calculated according to standard curve.

\subsubsection{Preparation of RIF microspheres by lyophilization}

Solutions of RIF were prepared by weighting excess RIF into $5 \mathrm{~g}$ of different ratio $(0,20 \%, 45 \%$ and $55 \%, 70 \%, 100 \%$ ) of TBA solution followed by sonication, then filtered through $0.22 \mu \mathrm{m}$ filter to remove undissolved solid. The clear, saturated solution was filled into vials and lyophilized using the followed cycle (Advantage 2.0 EL Lyophilizer): 1. Thermal treatment: ramp the temperature to $-20^{\circ} \mathrm{C}$ and hold for 2 hours, and then further reduce the temperature to $40{ }^{\circ} \mathrm{C}$ and hold for another 2 hours; 2 . Primary drying: first turn on the vacuum to $350 \mathrm{mTorr}$ and slowly ramp the temperature to $-20^{\circ} \mathrm{C}$ in 2 hours, hold this temperature for 10 hours; and then increase the vacuum to $300 \mathrm{mTorr}$ and increase the temperature to $0^{\circ} \mathrm{C}$, and dry for 2 hours; 3 . 
Secondary drying: Further increase the vacuum to 200 mTorr, and increase the temperature to 25

${ }^{\circ} \mathrm{C}$, and dry for 4 hours.

\subsubsection{Scanning electron microscopy (SEM)}

Lyophilized RIF microspheres were mounted onto aluminum stubs using double sided adhesive tape, and sputter coated with a thin layer of gold. A SEM (FEI Quanta 200, Netherlands) was used to study the morphology and size of the lyophilized RIF microspheres, using an acceleration potential of $15 \mathrm{kV}$.

\subsubsection{Dynamic light scattering (DLS)}

The size distribution of the lyophilized RIF microspheres (in dry powder form) was measured using a dry powder laser light scattering method (Malvern Mastersizer 2000 with accessory system Scirocco 2000 (A), Malvern Instruments, Malvern, UK). Particle size distribution is characterized by the mass median diameter (D10, D50 and D90) and the Volume Weighted Mean Diameter. Three measurements were performed and the average value was calculated.

\subsubsection{Powder X-ray diffraction (PXRD)}

The various RIF microspheres were measured at room temperature by a X' pert Powder (PANalytical, $\mathrm{X}^{\prime}$ Pert Powder) equipped with $\mathrm{Cu} \mathrm{K} \alpha$ radiation and scanned from 5 to $50^{\circ}(2 \theta)$ with a step size of $0.0016^{\circ}$. The scan rate was $1 \%$ min.

\subsubsection{In vitro infection and treatment}

The human monocyte cell line THP-1 cells was cultured in RPMI 1640 medium supplemented with $10 \% \mathrm{FBS}$ and $1 \%$ streptomycin and penicillin. Culture plates were maintained at $37^{\circ} \mathrm{C}$ and $5 \%$ $\mathrm{CO}_{2}$. Cells were seeded at a density of $2 \times 10^{5}$ cells/well in 6-well plates and were induced to differentiate into macrophages by incubation in the presence of $20 \mathrm{nM}$ phorbol myristate acetate (PMA) for $14 \mathrm{~h}$. Cells were then washed and incubated in fresh medium (without antibiotic) for 
another $10 \mathrm{~h}$. Lyophilized RIF microspheres were suspended in $2 \mathrm{~mL}$ of RPMI 1640 at a final concentration of $15 \mu \mathrm{g} / \mathrm{mL}$ and then the suspension was added into the cell culture medium. The 6-well plates were observed under microscope (Carl Zeiss, Germany) after 15 minutes.

\section{Results and Discussion}

\subsection{Solubility of crystalline RIF in various ratios of TBA/water cosolvent}

RIF (Fig. 1) has two crystal forms (Form I and Form II), and several solvates forms (Becker et al., 2009; Henwood* et al., 2001; Pelizza et al., 1977). In this experiment, the RIF raw material is crystal Form I. RIF is a hydrophobic compound with a $\log \mathrm{P}$ value reported to be 2.7 to 4.2 under different $\mathrm{pH}$ (Becker et al., 2009). In order to identify a suitable range of TBA/water ratio that is amendable for RIF lyophilization, we first determined the solubility of crystalline RIF (Form I) in various ratios of TBA/water cosolvent, summarized in Tab. 1. Apparently, RIF achieved best solubility of $>60 \mathrm{mg} / \mathrm{mL}$ in $\sim 50-65 \%$ TBA/water cosolvent, while its solubility in either pure water or pure TBA is much lower, at 0.41 or $14 \mathrm{mg} / \mathrm{mL}$, respectively (Tab. 1). From the perspective of maximizing the manufacturing yield, 50-65\% TBA/water certainly is most suitable for lyophilization of RIF. In the next section, we also observed that the morphology of the RIF lyophile is dictated by the TBA/water ratio.

\subsection{Morphology and size distribution of RIF microspheres prepared by lyophilization}

RIF was dissolved in different ratios of TBA/water cosolvent, lyophilized, and then observed with SEM (Fig. 2). Very interestingly, uniform spherical RIF particles were obtained using 20\%, $45 \%$, and $55 \% \mathrm{TBA} /$ water cosolvent while not with pure water (0\% TBA), $70 \%$ TBA, or pure 
TBA. The size of microspheres prepared by $20 \%$ TBA appears to be the largest, around $6-7 \mu \mathrm{m}$; while those by $45 \%$ or $55 \%$ TBA are much smaller, around $1-3 \mu \mathrm{m}$.

The size distribution of microspheres prepared by 55\% TBA solution was further characterized by laser light scattering (Fig. 3). The RIF microspheres have D10, D50 and D90 values of 0.627, 1.622, and $5.218 \mu \mathrm{m}$, respectively, and a volume-weighted mean diameter of $2.376 \mu \mathrm{m}$. This narrow size distribution around $1-3 \mu \mathrm{m}$ is especially suitable for pulmonary inhalation for anti-TB therapy. As demonstrated by several experimental (Davies et al., 1972; Heyder et al., 1975; Muir and Davies, 1967; Winchester et al., 1984) and theoretical (Clark and Egan, 1994; Gerrity et al., 1979; Heyder and Rudolf, 1984) studies, this size range could facilitate a large portion of microspheres to be deposited into the deep lung (alveolar region). As widely known, very large particles (aerodynamic diameter $>10 \mu \mathrm{m}$ ) have little chance of leaving the mouth after inhalation, and relatively large particles $(8-10 \mu \mathrm{m})$ deposit mostly in the trachea and bronchus, while very small particles $(<1 \mu \mathrm{m})$ will be mostly exhaled (Edwards et al., 1998). Furthermore, particle size 1-3 $\mu \mathrm{m}$ might also enhance AMФ phagocytosis (Edwards et al., 1998), which will be demonstrated by the later macrophage phagocytosis study.

\subsection{Crystallinity of lyophilized RIF microspheres}

We used powder x-ray diffraction to study the crystallinity of the lyophilized RIF microspheres. As shown in Fig. 4, the absence of diffraction peaks from any lyophilized RIF microsphere clearly indicated the amorphous nature of these RIF microspheres. The amorphous RIF particles are advantageous over crystalline RIF particles as potential inhalation therapy for anti-TB therapy, since amorphous drugs are well-known to have much higher solubility than its crystalline counterpart. The solubility ratio of the amorphous vs. the crystalline form at any given 
temperature (T) is directly related with the free energy difference ( $\Delta \mathrm{G}$ ) between those two forms (Hancock and Parks, 2000):

$$
\Delta \mathrm{G}_{T}^{A_{i} C}=-R T \ln \frac{a_{\text {amorphous }}}{a_{\text {erystal }}}
$$

Where $a$ is activity of the solute in the saturated solution, which in dilute solution where activity coefficients are essentially unity, is the concentration at saturation or the solubility, $\mathrm{R}$ is the gas constant. When only the effect of water sorption is ignored, the theoretically estimated solubility enhancement ratio of amorphous to crystal form is 9.9 (Murdande et al., 2010).

Pharmacologically, the anti-TB effect of rifampicin is due to its high affinity binding and the inhibition of the bacterial DNA-dependent RNA polymerase (Hartmann et al., 1967). Some research has demonstrated that local high concentration of RIF enhanced its diffusion into the alveolus region and improved the sterilization, at least in short-term (Diacon et al., 2007; Ruslami et al., 2007). Given a 10 time increased solubility, the amorphous RIF microspheres theoretically could provide a concentration gradient $~ 10$ time higher to greatly facilitate the drug penetration, and generate a much higher RIF concentration in the alveolus to eradicate the Mtb.

\subsection{Formation mechanism of RIF microspheres: freezing induced LLPS.}

To understand the formation mechanism of the RIF microspheres during lyophilization, it is worthwhile to re-visit the binary phase diagram of the TBA/water system that was reported by Kasraian et al. (Fig. 5) (Kasraian and DeLuca, 1995). For three ratios of RIF TBA/water solution (20\%, 45\%, and 55\% TBA) that produced RIF microsphere upon lyophilization, the initial RIF solutions were saturated (refer to Table 1 for saturated concentration). Freezing of $20 \%$ TBA solution, and $45 \%$ or $55 \%$ TBA solution was different in terms of form transition process: when 
frozen, the $20 \%$ TBA solution underwent a single step phase transition, i.e., from a liquid solution ( $\mathrm{A}_{1}$ step, as $20 \%$ TBA solution) to a solid mixture ( $\mathrm{A}_{2}$ step, as eutectic solid $\left.\mathrm{A}\right)$ as illustrated as Fig. 5; while when $45 \%$ or $55 \%$ TBA solution was frozen, the TBA solution underwent a two-step phase transition process, i.e., from liquid ( $\mathrm{B}_{1}$ step, as $45 \%$ or $55 \%$ TBA solution) to a mixture of liquid/solid ( $\mathrm{B}_{2}$ step, TBA hydrate + TBA solution with decreasing TBA content), and then to solid phase ( $\mathrm{B}_{3}$ step, TBA hydrate + eutectic solid $\left.\mathrm{A}\right)$.

We hypothesized that RIF microspheres was formed in $A_{1}$ step 1 for RIF solution in $20 \%$ TBA/water, and in $\mathrm{B}_{1}$ and/or $\mathrm{B}_{2}$ steps for $\mathrm{RIF}$ solution in $45 \%$ or $55 \% \mathrm{TBA} / \mathrm{water}$. This is because, when the solution is completely frozen into crystalline solids $\left(A_{2}\right.$ or $B_{3}$ step), the molecular mobility would be too low in crystalline TBA hydrate or ice to allow the rearrangement of molecules to form spherical structures.

Slowly freezing of a saturated RIF solution in $20 \% \mathrm{TBA} /$ water solution from room temperature to $-5^{\circ} \mathrm{C}$ (the eutectic melting temperature), the solubility of RIF is expected to decrease and thus, supersaturation could occur. In an initially homogenous solution of water, TBA, and RIF, three potential scenarios could occur once RIF is supersaturated depending on the extent of supersaturation and the physicochemical properties of RIF:

Scenario 1: Remain as a supersaturated homogenous solution. No phase separation happens until the eutectic melting point $\left(-5^{\circ} \mathrm{C}\right)$ is reached, and RIF distributes homogenously within the crystal boundaries of the frozen eutectic solids. The lyophile obtained after the sublimation of the solids would not display any ordered morphological features. This is certainly not the case for RIF lyophilized with $20 \%$ TBA;

Scenario 2: RIF starts to crystallize once its TBA/water solution is supersaturated, thus to decrease the solution concentration. If this occurred in the RIF solution in the $20 \%$ TBA/water, 
the lyophilized RIF particles would be crystalline. Clearly, this again was not what had happened since the RIF particles obtained were all amorphous (Fig. 4).

Scenario 3: LLPS occurs in the TBA/water solution, where two liquid phases appear, with one rich in RIF and TBA (the "oil" phase), while the other phase is largely water (the "water" phase), and an oil-in-water emulsion could form due to the surface tension. The emulsion structure could be frozen and spherical particles could form upon the sublimation of the solids. Based on the SEM pictures, this appears to be a plausible mechanism for RIF microsphere formation upon lyophilizing with a 20\% TBA/water.

As a comparison, slow freezing of a saturated RIF solution in 45 or $55 \% \mathrm{TBA} / \mathrm{water}$ solution from room temperature to $-5{ }^{\circ} \mathrm{C}$ could produce RIF supersaturation with two mechanisms: first, similarly as the $20 \%$ TBA/water solution, the decreasing of temperature could reduce the solubility of RIF ( $B_{1}$ step in Fig. 5); and second, during step $B_{2}$ (Fig. 5), the TBA contents deceases with the further decrease of temperature, as TBA hydrate precipitates, and we have demonstrated that the solubility of RIF in TBA/water decreases rapidly with the decrease of TBA content (Table 1). This dual-mechanism could generate a high extent of RIF supersaturation when freezing a $45 \%$ or $55 \%$ TBA/water solution, thus to cause a fast LLPS similarly as the fast nucleation process at high supersaturation, thus, therefore, smaller particle size could be obtained when 45 or $55 \%$ of TBA/water was used (Fig. 2).

The LLPS of RIF solution in TBA/water could be directly observed under POM. As we cooled the temperature of a saturation solution of RIF in $55 \% \mathrm{TBA} /$ water from room temperature to $4{ }^{\circ} \mathrm{C}$ in a lab refrigerator, the solution became turbid with visible precipitates. When observed under POM (Fig. 6), the precipitates appeared to be transparent, amorphous, and uniform droplets with 
diameter of about $2 \mu \mathrm{m}$. Once frozen and lyophilized, these droplets could eventually be converted into amorphous RIF microspheres.

\subsection{Lyophilization of four other drugs using TBA/water}

In order to investigate if this mechanism of freezing induced LLPS could produce similar uniform microspheres of other drugs, we dissolved four other drugs, i.e., rifaximin, rifapentine, paclitaxel, and isoniazid (Fig. 7) in 55\% TBA/water solution to obtain saturation, and then lyophilized the saturated solution similarly as RIF. As observed under SEM (Fig. 8), among these four different drugs, rifaximin and paclitaxel (Fig. 8, A and C) could form microspheres upon the lyophilization of their TBA/water solution, while rifapentine and isoniazid (Fig. 8, B and D) formed irregular and fibrillar, network-like structure after the same lyophilization cycle. Note that RIF, rifaximin and rifapentine are structurally related, while paclitaxel and isoniazid are structurally independent. The SEM morphology of these lyophiles suggests that the underlying mechanism of uniform microsphere formation is dependent mostly on the physicochemical properties (e.g., partition condition of the drug, solubility of the drug in water and organic solvent, etc.) such as partition condition of the drug in oil and water while not on their chemical structure. Therefore, this approach could potentially be used as a particle engineering tool for compounds with a suitable set of physiochemical characteristics, which need to be further determined and defined. Although more systemic investigation is needed, we hypothesize that the ternary phase behavior of drug-TBA-water at different temperature, and the crystallization tendency of the drug, could significantly influence the morphology of the lyophile obtained from the TBA/water system. 


\subsection{Phagocytosis of RIF microspheres by macrophages.}

To be able to generate a fast pharmacological effect against Mtb after being inhaled into the alveolar space, the RIF microspheres have to be phagocytosed by АMФs rapidly. We evaluated the phagocytosis process of RIF microsphere by macrophages in a cell culture study. In this evaluation, a human monocyte cell line, THP-1 cells, were induced to differentiate into macrophages by incubation in the presence of phorbol myristate acetate (PMA). The lyophilized RIF microspheres were added into the cell culture and observed under microscope. After 15 minutes, intensive phagocytosis could be clearly observed under the inverted microscope (Fig. 9). In fact, some of the macrophages (in red circles) broke and die due to overwhelming amount of microsphere uptake. Combined with the higher kinetic solubility of amorphous RIF and suitable size distribution, these properties of the lyophilized RIF microspheres demonstrate them to be potentially highly effective inhalation therapies for anti-TB application.

\section{Conclusions}

We demonstrated that uniform and amorphous RIF microspheres could be obtained through lyophilization of a TBA/water solution, a routine industrial manufacturing process that is widely accessible and readily scalable. The obtained RIF microspheres have a mono-dispersive particle size distribution between 1-3 $\mu \mathrm{m}$, a high RIF solubility, and could be phagocytized readily by macrophages. All these properties are highly desirable for a potential inhalation therapy targeting Mtb residing АМФs. We conclude that the RIF microspheres formation is caused by LLPS occurred within the TBA/water solution upon cooling and freezing during lyophilization, and this mechanism could be used to produce drug microspheres of a range APIs (active pharmaceutical ingredients) with suitable physicochemical properties. 
It's certainly worthwhile to emphasize that a successful inhalation formulation requires more than just a drug particle with suitable particle size distribution. Other API particle properties, excipient (typically lactose, lecithin) properties, and the selection of the dry powder inhaler (DPI), etc., are all critically important (Misra et al., 2011; Telko and Hickey, 2005) to ensure the efficient delivery of drugs into the deep lung. At the same time, the level of residual TBA within the lyophilized RIF particles has to be controlled to ensure safety and to meet regulatory requirements. Investigations have been carried out by other researchers to understand the impact of factors such as initial TBA concentration, freeze-drying conditions, and cake thickness, etc., on the level of residual TBA within the lyophiles. (Wittaya-Areekul S et al., 1998; WittayaAreekul S et al., 2002). These related investigations will be continued in our lab to evaluate the application of RIF amorphous microsphere as an inhaled medicine.

\section{Acknowledgement}

This research is supported by Bill \& Melinda Gates Foundation Grand Challenges Explorations (GCE) Grant, Grant Number: OPP1021992. F Qian also thanks for the start-up funds provided by the Center for Life Sciences at Tsinghua and Peking Universities (Beijing, China), and by the China Recruitment Program of Global Experts. 


\section{References}

Becker, C., Dressman, J.B., Junginger, H.E., Kopp, S., Midha, K.K., Shah, V.P., Stavchansky, S., Barends, D.M., 2009. Biowaiver monographs for immediate release solid oral dosage forms: Rifampicin. Journal of Pharmaceutical Sciences 98, 2252-2267.

Blasi, P., Schoubben, A., Giovagnoli, S., Rossi, C., Ricci, M., 2009. Fighting tuberculosis: old drugs, new formulations. Expert Opinion on Drug Delivery 6, 977-993.

Champion, J., Walker, A., Mitragotri, S., 2008. Role of particle size in phagocytosis of polymeric microspheres. Pharmaceutical Research 25, 1815-1821.

Chhabria, M., Jani, M., Patel, S., 2009. New frontiers in the therapy of tuberculosis: fighting with the global menace. Mini Reviews in Medicinal Chemistry 9, 401-430.

Chow, A.L., Tong, H.Y., Chattopadhyay, P., Shekunov, B., 2007. Particle engineering for pulmonary drug delivery. Pharmaceutical Research 24, 411-437.

Clark, A.R., Egan, M., 1994. Modelling the deposition of inhaled powdered drug aerosols. Journal of Aerosol Science 25, 175-186.

Davies, C.N., Heyder, J., Subba Ramu, M.C., 1972. Breathing of half-micron aerosols. I. Experimental. Journal of Applied Physiology 32,591-600.

de Waard, H., Hinrichs, W.L.J., Frijlink, H.W., 2008. A novel bottom-up process to produce drug nanocrystals: Controlled crystallization during freeze-drying. Journal of Controlled Release 128, 179-183.

Diacon, A.H., Patientia, R.F., Venter, A., van Helden, P.D., Smith, P.J., McIlleron, H., Maritz, J.S., Donald, P.R., 2007. Early bactericidal activity of high-dose rifampin in patients with pulmonary tuberculosis evidenced by positive sputum smears. Antimicrobial Agents and Chemotherapy 51, 2994-2996. 
Dorman, S.E., Chaisson, R.E., 2007. From magic bullets back to the magic mountain: the rise of extensively drug-resistant tuberculosis. Nature Medicine 13, 295-298.

Edwards, D.A., Ben-Jebria, A., Langer, R., 1998. Recent advances in pulmonary drug delivery using large, porous inhaled particles. Journal of Applied Physiology 85, 379-385.

Gerrity, T.R., Lee, P.S., Hass, F.J., Marinelli, A., Werner, P., Lourenco, R.V., 1979. Calculated deposition of inhaled particles in the airway generations of normal subjects. Journal of Applied Physiology: Respiratory, Environmental and Exercise Physiology 47,867-73.

Ginsberg, A.M., 2010. Tuberculosis drug development: progress, challenges, and the road ahead. Tuberculosis 90, 162-167.

Hancock, B., Parks, M., 2000. What is the true solubility advantage of the different forms. Pharmaceutical Research 17, 397-404.

Hartmann, G., Honikel, K.O., Knüsel, F., Nüesch, J., 1967. The specific inhibition of the DNAdirected RNA synthesis by rifamycin. Biochimica et Biophysica Acta (BBA)-Nucleic Acids and Protein Synthesis 145, 843-844.

Henwood*, S.Q., Liebenberg, W., Tiedt, L.R., Lötter, A.P., de Villiers, M.M., 2001. Characterization of the solubility and dissolution properties of several new rifampicin polymorphs, solvates, and hydrates. Drug Development and Industrial Pharmacy 27, 1017-1030. Heyder, J., Armbruster, L., Gebhart, J., Grein, E., Stahlhofen, W., 1975. Total deposition of aerosol particles in the human respiratory tract for nose and mouth breathing. Journal of Aerosol Science 6, 311-328.

Heyder, J., Rudolf, G., 1984. Mathematical models of particle deposition in the human respiratory tract. Journal of Aerosol Science 15, 697-707. 
Kasraian, K., DeLuca, P., 1995. Thermal analysis of the tertiary butyl alcohol-water system and its implications on freeze-drying. Pharmaceutical Research 12, 484-490.

Kucuk I, Ahmad Z, Edirisinghe M, Orlu-Gul M, 2014. Utilization of microfluidic V-junction device to prepare surface itraconazole adsorbed nanospheres. International Journal of Pharmaceutics 472,339-346.

L. Remmele, R., Krishnan, S., J. Callahan, W., 2012. Development of stable lyophilized protein drug products. Current Pharmaceutical Biotechnology 13, 471-496.

Makino, K., Yamamoto, N., Higuchi, K., Harada, N., Ohshima, H., Terada, H., 2003. Phagocytic uptake of polystyrene microspheres by alveolar macrophages: effects of the size and surface properties of the microspheres. Colloids and Surfaces B: Biointerfaces 27, 33-39.

M. Enayati, Z. Ahmad, E. Stride , M. Edirisinghe, 2010. Size mapping of electric field-assisted production of polycaprolactone particles. Journal of The Royal Society Interface 7, S393-S402. Misra, A., Hickey, A.J., Rossi, C., Borchard, G., Terada, H., Makino, K., Fourie, P.B., Colombo, P., 2011. Inhaled drug therapy for treatment of tuberculosis. Tuberculosis 91, 71-81.

Mitchison, D.A., 2000. Role of individual drugs in the chemotherapy of tuberculosis. The International Journal of Tuberculosis and Lung Disease 4, 796-806.

Muir, D.C.F., Davies, C.N., 1967. The deposition of $0.5 \mu \mathrm{m}$ diameter aerosols in the lungs of man. Annals of Occupational Hygiene 10, 161-174.

Murdande, S.B., Pikal, M.J., Shanker, R.M., Bogner, R.H., 2010. Solubility advantage of amorphous pharmaceuticals: I. A thermodynamic analysis. Journal of Pharmaceutical Sciences 99, 1254-1264.

Muttil, P., Wang, C., Hickey, A., 2009. Inhaled drug delivery for tuberculosis therapy. Pharmaceutical Research 26, 2401-2416. 
Nangrejo M, Ahmad Z, Stride E, Edirisinghe M, Colombo P.,2008. Preparation of polymeric and ceramic porous capsules by a novel electrohydrodynamic process. Pharmceutical Development. and Technology 13,425-432.

Overhoff, K.A., Engstrom, J.D., Chen, B., Scherzer, B.D., Milner, T.E., Johnston, K.P., Williams Iii, R.O., 2007. Novel ultra-rapid freezing particle engineering process for enhancement of dissolution rates of poorly water-soluble drugs. European Journal of Pharmaceutics and Biopharmaceutics 65, 57-67.

Pelizza, G., Nebuloni, M., Ferrari, P., Gallo, G.G., 1977. Polymorphism of rifampicin. Farmaco Edizione Scitifica 32, 471-481.

Qian, F., Ni, N., Chen, J.-W., Desikan, S., Naringrekar, V., Hussain, M., Barbour, N., Smith, R., 2008. Formation of Zinc-Peptide Spherical Microparticles During Lyophilization from tert-Butyl Alcohol/Water Co-solvent System. Pharmaceutical Research 25, 2799-2806.

Rastogi, R., Sultana, Y., Ali, A., Aqil, M., 2006. Particulate and vesicular drug carriers in the management of tuberculosis. Current Drug Delivery 3, 121-128.

Ruslami, R., Nijland, H.M.J., Alisjahbana, B., Parwati, I., van Crevel, R., Aarnoutse, R.E., 2007. Pharmacokinetics and tolerability of a higher rifampin dose versus the standard dose in pulmonary tuberculosis patients. Antimicrobial Agents and Chemotherapy 51, 2546-2551.

Russell, D.G., 2007. Who puts the tubercle in tuberculosis? Nature Reviews Microbiology 5, 3947.

Seville, P.C., Li, H.-y., Learoyd, T.P., 2007. Spray-dried powders for pulmonary drug delivery. Critical Reviews in Therapeutic Drug Carrier Systems 24, 307-360.

Shoyele, S., 2008. Engineering protein particles for pulmonary drugdelivery, in: Jain, K. (Ed.), Drug Delivery Systems. Humana Press, pp. 149-160. 
Shoyele, S.A., Cawthorne, S., 2006. Particle engineering techniques for inhaled biopharmaceuticals. Advanced Drug Delivery Reviews 58, 1009-1029.

Telko, M.J., Hickey, A.J., 2005. Dry Powder Inhaler Formulation. Respiratory Care 50, 12091227.

Walkey, C.D., Olsen, J.B., Guo, H., Emili, A., Chan, W.C.W., 2012. Nanoparticle Size and Surface Chemistry Determine Serum Protein Adsorption and Macrophage Uptake. Journal of the American Chemical Society 134, 2139-2147.

Wang, W., 2000. Lyophilization and development of solid protein pharmaceuticals. International Journal of Pharmaceutics 203, 1-60.

Wattendorf, U., Koch, M.C., Walter, E., Vörös, J., Textor, M., Merkle, H.P., 2006. Phagocytosis of poly(L-lysine)-graft-poly (ethylene glycol) coated microspheres by antigen presenting cells: Impact of grafting ratio and poly (ethylene glycol) chain length on cellular recognition. Biointerphases 1, 123-133.

Winchester, J.W., Jones, D.L., Mu-tian, B., 1984. Aerosol deposition in the human respiratory tract. Nuclear Instruments and Methods in Physics Research Section B: Beam Interactions with Materials and Atoms 3, 360-363.

Wittaya-Areekul S, Nail SL.,1998. Freeze-drying of tert-butyl alcohol/water cosolvent systems: effects of formulation and process variables on residual solvents. Journal of Pharmaceutical Sciences 87, 491-495.

Wittaya-Areekul S, Needham GF, Milton N, Roy ML, Nail SL., 2002. Freeze-drying of tertbutanol/water cosolvent systems: a case report on formation of a friable freeze-dried powder of tobramycin sulfate. Journal of Pharmaceutical Sciences 91, 1147-1155. 
Yoo, J.-W., Doshi, N., Mitragotri, S., 2011. Adaptive micro and nanoparticles: temporal control over carrier properties to facilitate drug delivery. Advanced Drug Delivery Reviews 63, 12471256. 
Table 1. Solubility of crystalline RIF (Form I) in various ratios of TBA/water cosolvent $\left(37^{\circ} \mathrm{C}\right)$

\begin{tabular}{cccccccccccccc}
\hline TBA (wt. \%) & 0 & 20 & 30 & 35 & 40 & 45 & 50 & 55 & 60 & 65 & 70 & 80 & 100 \\
$\mathrm{RIF}(\mathrm{mg} / \mathrm{ml}) *$ & 0.41 & 10.53 & 25.29 & 35.32 & 42.00 & 51.05 & 64.97 & 65.53 & 62.05 & 62.05 & 26.54 & 31.56 & 14.01 \\
\hline
\end{tabular}

* RIF concentration was determined by UV analysis of the saturated supernatant 


\section{Figure captions}

Figure 1. Chemical structure of rifampicin (RIF). Formula: $\mathrm{C}_{43} \mathrm{H}_{58} \mathrm{~N}_{4} \mathrm{O}_{12}$; Molecular weight: $822.94 \mathrm{~g} / \mathrm{mol}$; Solubility: $14.01 \mathrm{mg} / \mathrm{ml} ; T_{\mathrm{m}}: 183^{\circ} \mathrm{C} ; \log \mathrm{P}: 2.7$.

Figure 2. Scanning electron micrographs of the RIF lyophiles obtained using 0, 20\%, 45\%, 55\%, $70 \%$ and $100 \%$ TBA/water solutions.

Figure 3. Size distribution of the lyophilized RIF microspheres prepared with 55\% TBA solution.

Figure 4. PXRD of Form I crystalline RIF and lyophilized RIF microspheres.

Figure 5. Phase diagram of the TBA-water system. (Pharm Res (1995) 12:484-490.) Arrows 1, 2 and 3 represent different phase transitions during the freezing process of a $55 \% \mathrm{TBA} / \mathrm{water}$ solution. $\mathrm{x}$ : TBA concentration on mole fraction basis.

Figure 6. Polarizing microscope photograph of excess amorphous RIF in 55\% TBA solution. LLPS occurred and uniform microspheres with size about $2 \mu \mathrm{m}$ were observed during the airdrying.

Figure 7. Chemical structure and physicochemical properties of four drugs.

Figure 8. Scanning electron micrographs of the lyophilizates of Rifaximin (A), Rifapentine (B), Paclitaxel(C) and Isoniazid (D). All drugs were dissolved in 55\% of TBA solution.

Figure 9. In vitro macrophages phagocytosis of lyophilized RIF microspheres in PBS (phosphate buffered saline) containing $1 \%$ poloxamer188-20X-5. The red circles were typical results which illustrate that RIF microspheres (small black dots) were phagocytosed by macrophages (large white circles). 


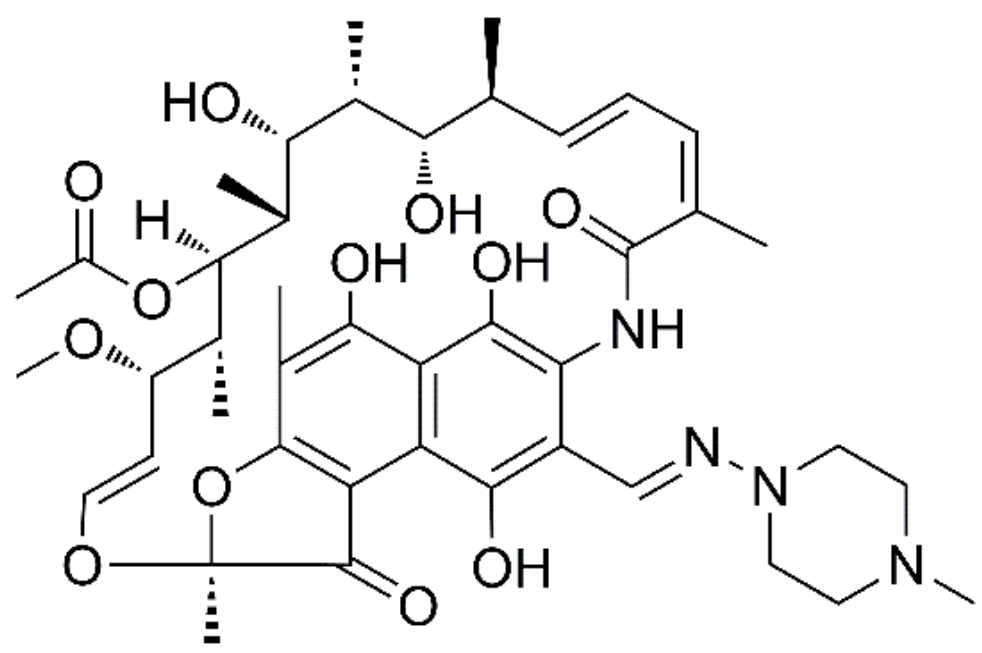

Figure 1 

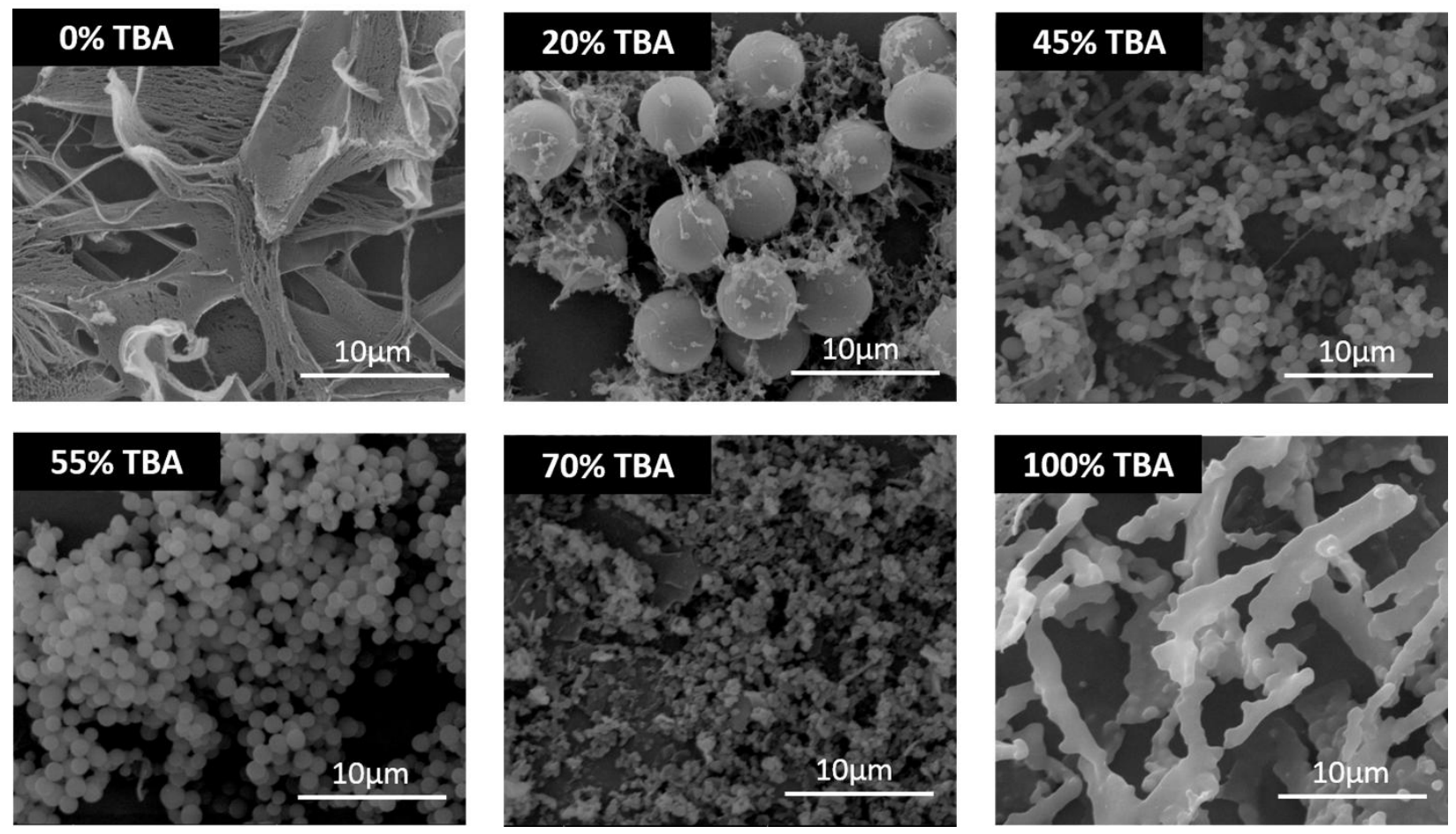

Figure 2 


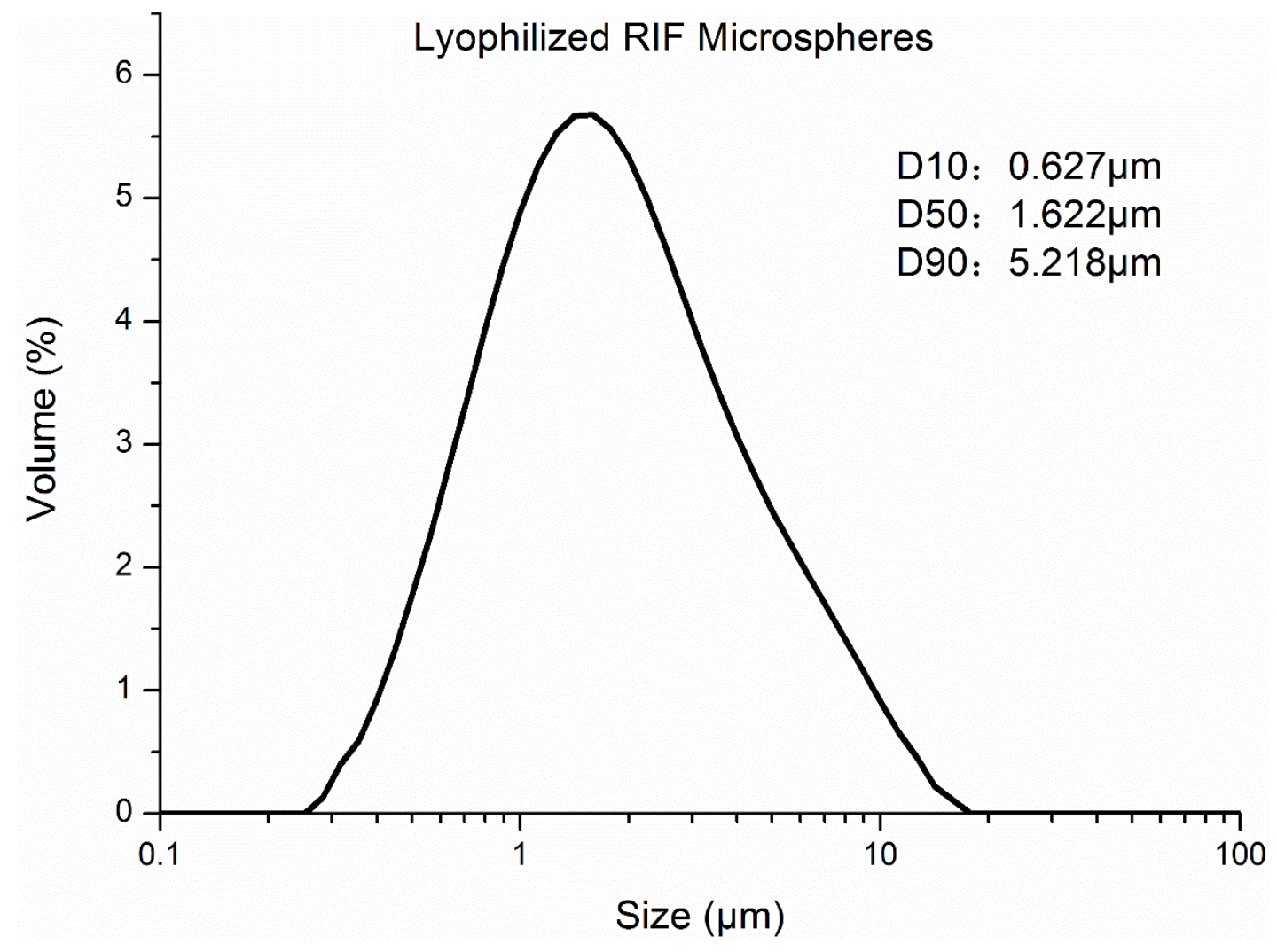

Figure 3 


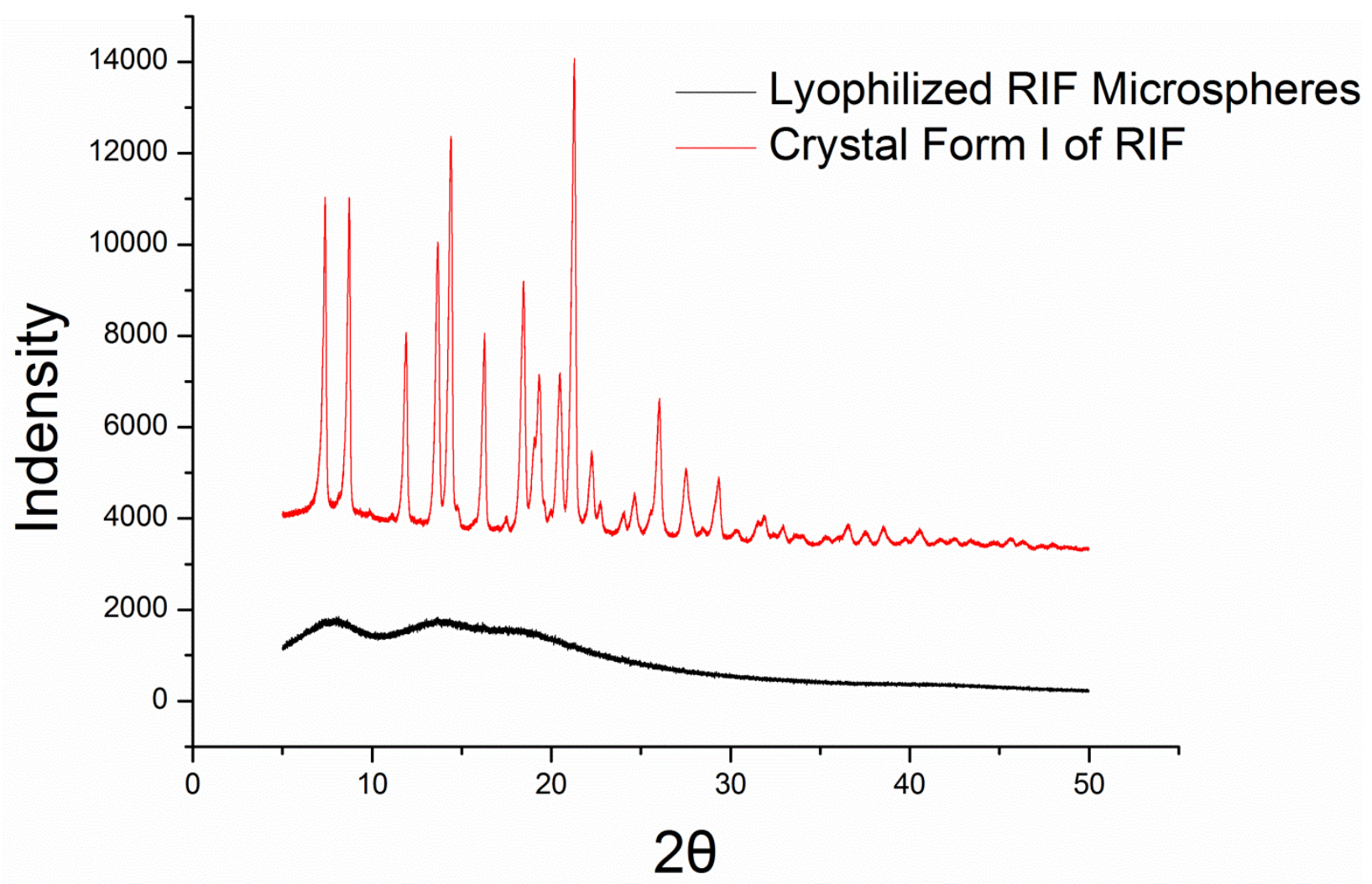

Figure 4 


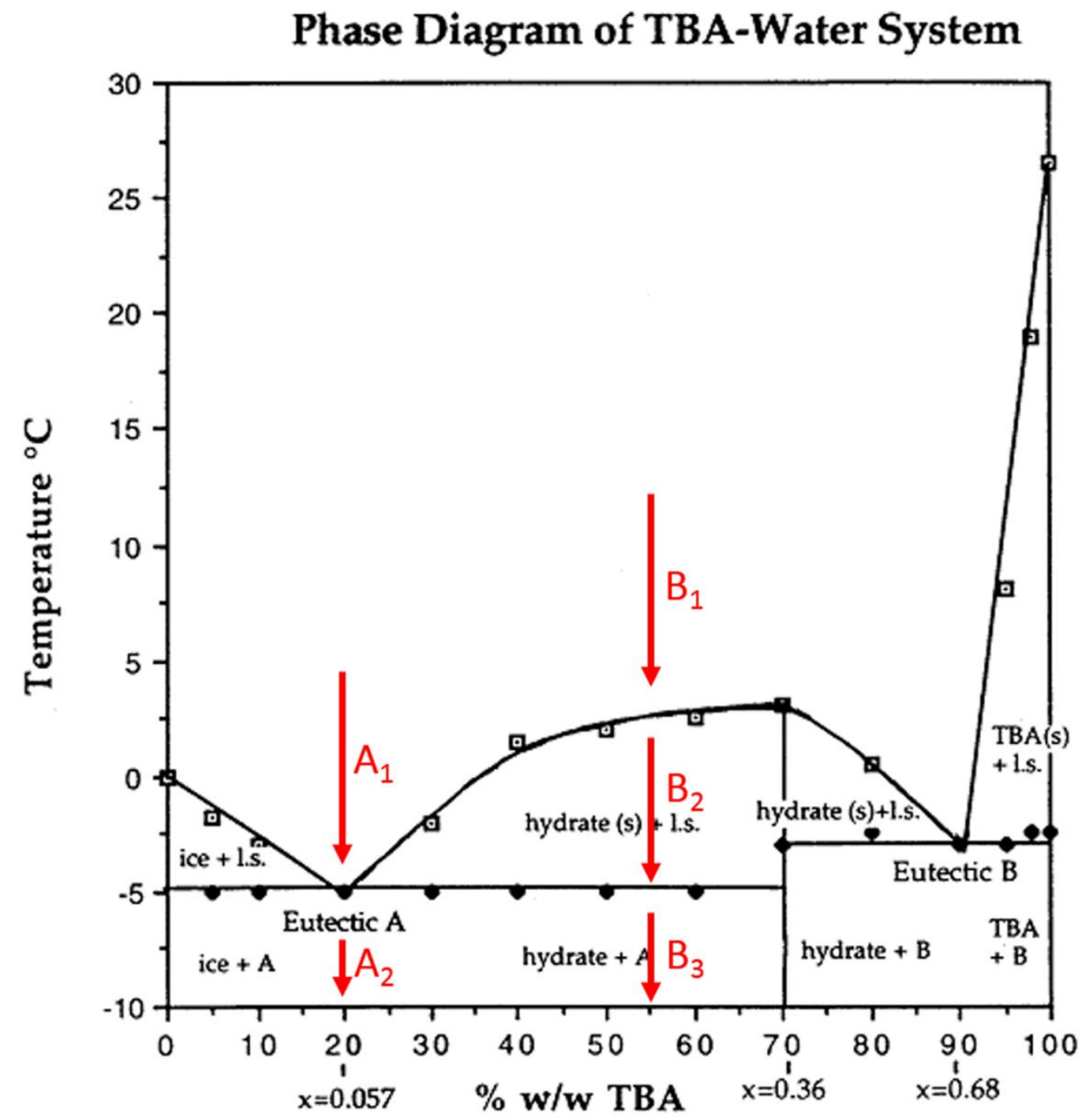

Figure 5 


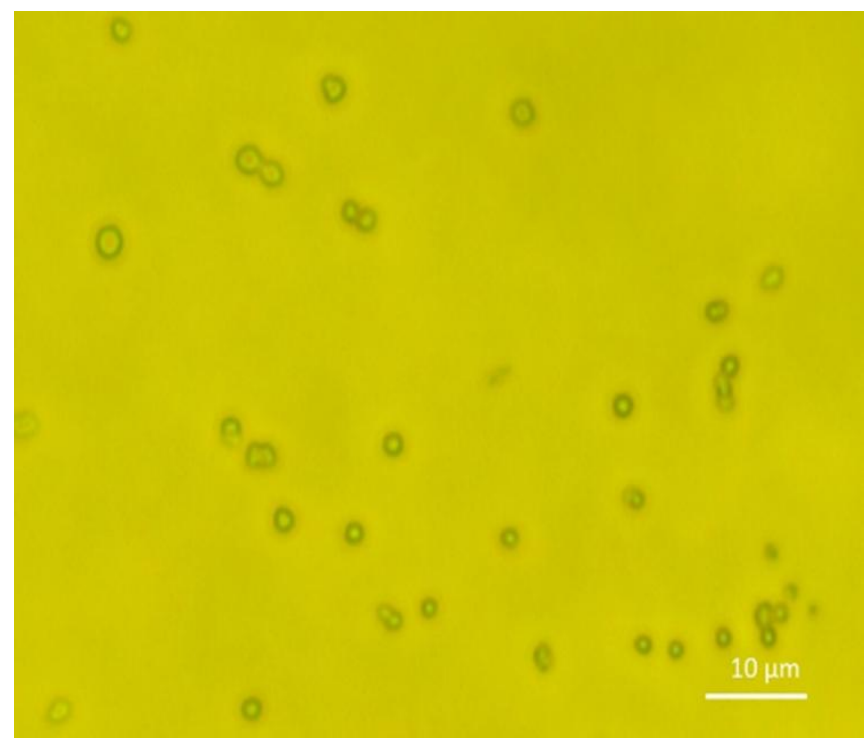

Figure 6 


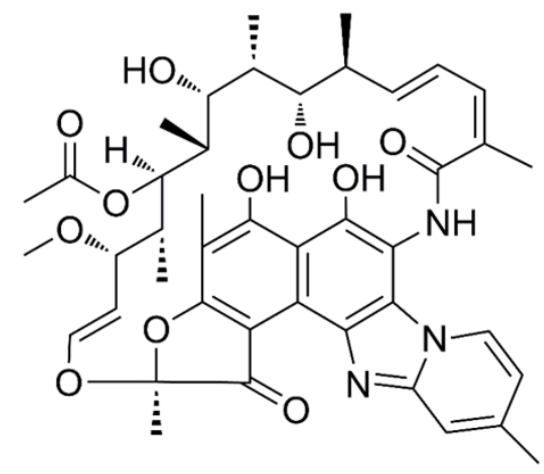

A. Rifaximin. Formula: $\mathrm{C}_{43} \mathrm{H}_{51} \mathrm{~N}_{3} \mathrm{O}_{11}$; Mw: $785.35 \mathrm{~g} / \mathrm{mol}$; $\log$ P: 2.6; Solubility: $7.38 \times 10^{-3} \mathrm{mg} / \mathrm{ml} ; T_{\mathrm{m}}: 218-228^{\circ} \mathrm{C}$;

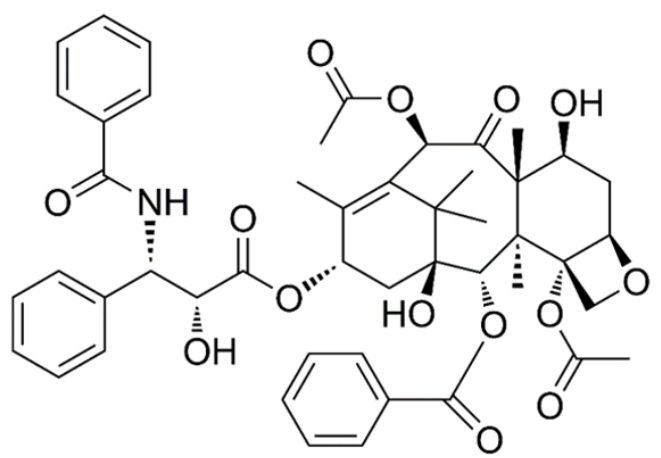

C. Paclitaxel. Formula: $\mathrm{C}_{47} \mathrm{H}_{51} \mathrm{NO}_{14} ; \mathrm{Mw}: 853.91 \mathrm{~g} / \mathrm{mol}$; $\log$ P: 3.5 ; Solubility: $3 \times 10^{-4} \mathrm{mg} / \mathrm{ml} ; T_{\mathrm{m}}: 216-217^{\circ} \mathrm{C}$;

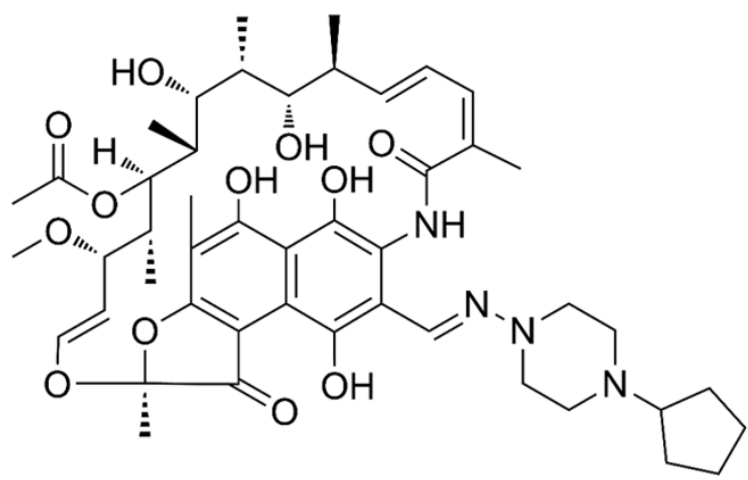

B. Rifapentine. Formula: $\mathrm{C}_{47} \mathrm{H}_{64} \mathrm{~N}_{4} \mathrm{O}_{12} ; \mathrm{Mw}: 876.45 \mathrm{~g} / \mathrm{mol}$; $\log$ P: 4; Solubility:2.13 × 10-2 $\mathrm{mg} / \mathrm{ml} ; T_{\mathrm{m}}: 179-180^{\circ} \mathrm{C}$;<smiles>NNC(=O)c1ccncc1</smiles>

D. Isoniazid. Formula: $\mathrm{C}_{6} \mathrm{H}_{7} \mathrm{~N}_{3} \mathrm{O} ; \mathrm{Mw}: 137.14 \mathrm{~g} / \mathrm{mol}$; $\log$ P: -0.7; Solubility: $140 \mathrm{mg} / \mathrm{ml} ; T_{\mathrm{m}}: 170-173^{\circ} \mathrm{C}$;

Figure 7 

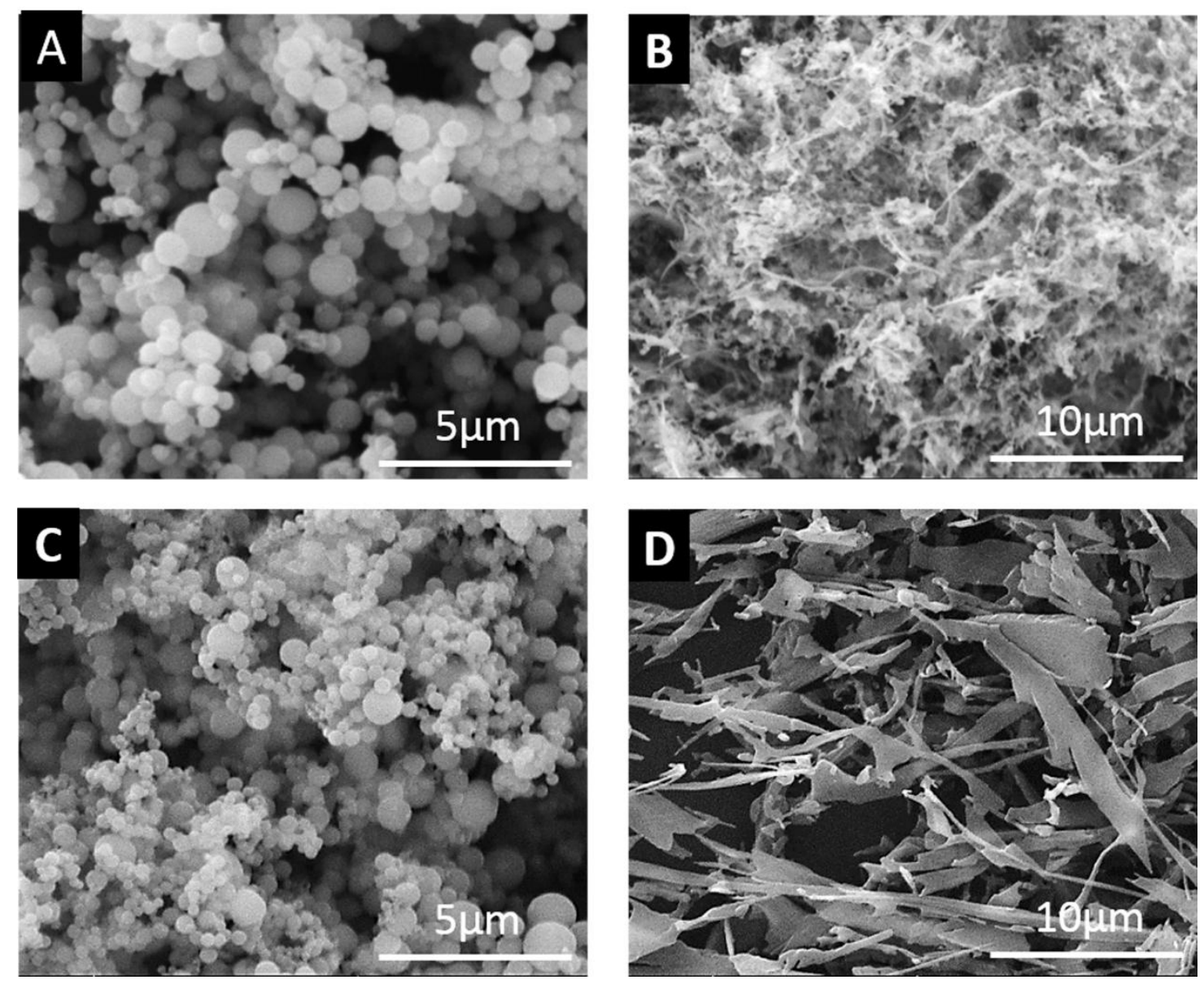

Figure 8 


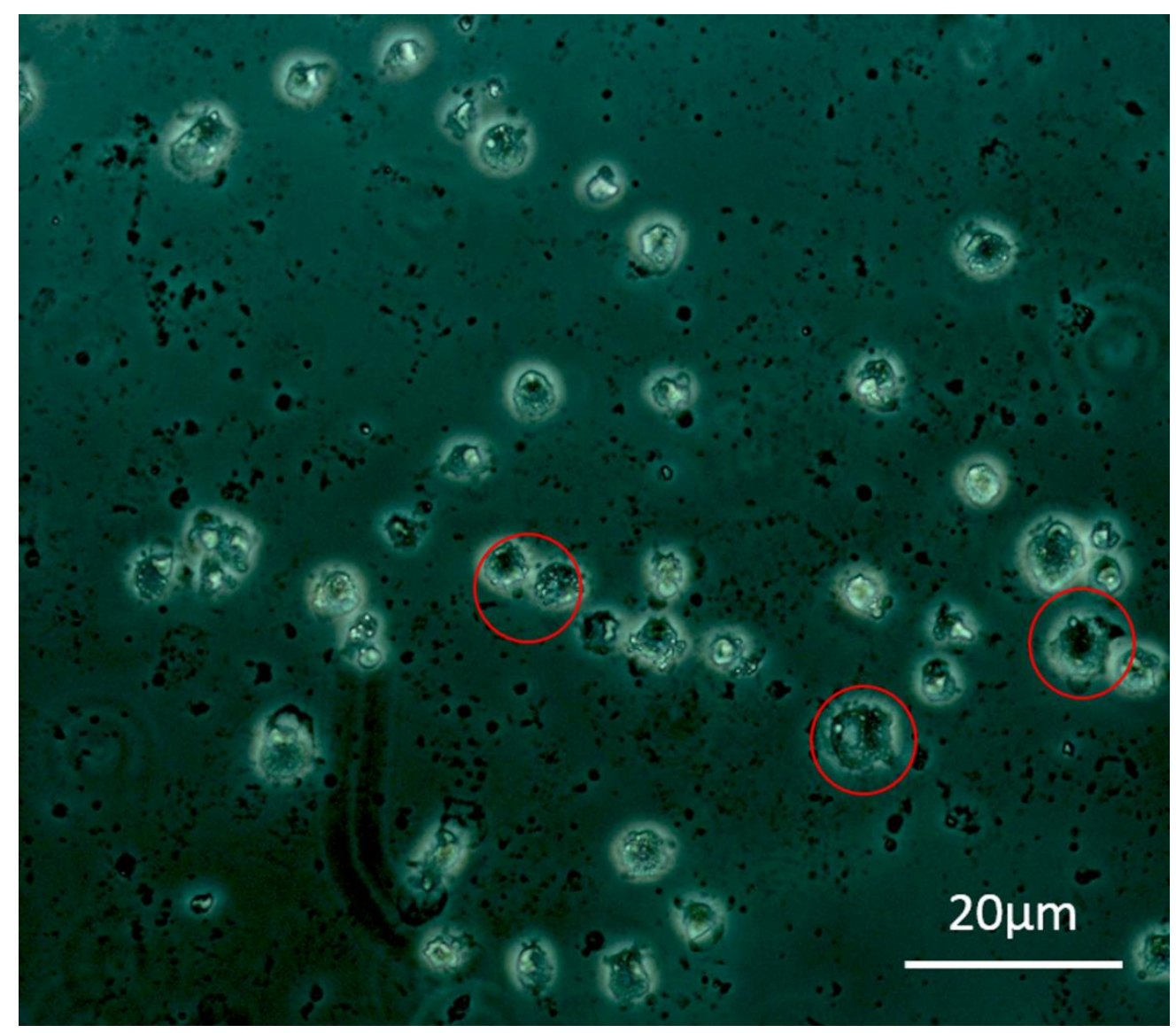

Figure 9 

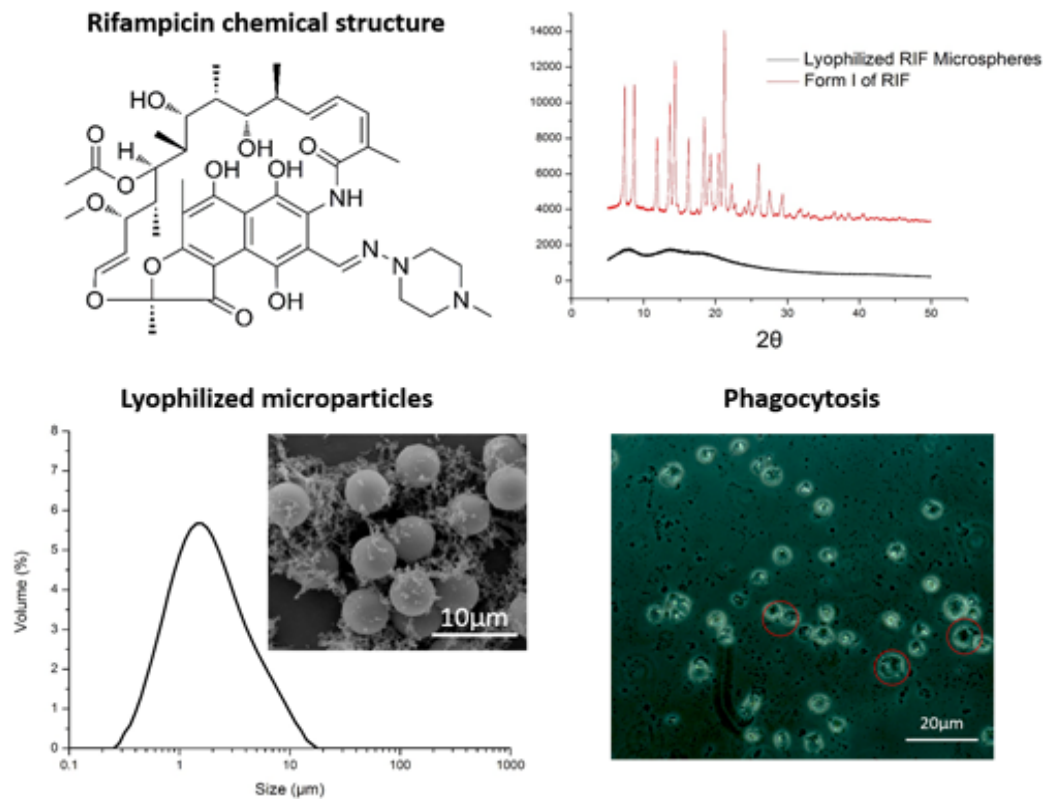